\title{
Exemplar Based Image Inpainting
}

\author{
Ankitha A \\ Department of Electronics \& Communication, St. Joseph Engineering College, Vamanjoor, Mangalore, \\ Karnataka, India
}

\begin{abstract}
A new algorithm is proposed for removing large objects from digital images. The challenge is to fill in the hole that is left behind in a visually plausible way. This has been addressed by two classes of algorithms: (i) "inpainting algorithms" for filling in small image gaps, and (ii) "super resolution" techniques" for creating one enhanced resolution image. This paper presents a novel and efficient algorithm that combines the advantages of these two approaches. We first note that exemplar-based texture synthesis contains the essential process required to replicate both texture and structure; the success of structure propagation, however, is highly dependent on the order in which the filling proceeds. We propose a best-first algorithm in which the confidence in the synthesized pixel values is propagated in a manner similar to the propagation of information in inpainting.
\end{abstract}

Key Words: exemplar-based inpainting framework, non parametric patch sampling.

\section{Introduction}

Inpainting is the process of reconstructing lost or deteriorated parts of images or videos. Image inpainting consists in recovering the missing or corrupted parts of an image so that the reconstructed image looks natural. Image inpainting refers to methods which consist of filling-in missing regions (holes) in an image.

The basic idea is simple. Replace the masked region with its neighbouring pixels so that it looks like the neighbourhood. Several algorithms were designed. First method is the inpainting algorithm. Consider a region in the image to be inpainted. Algorithm starts from the boundary of this region and goes inside the region gradually filling everything in the boundary first. It takes a small neighbourhood around the pixel on the neighbourhood to be inpainted. This pixel is replaced by normalized weighted sum of all the known pixels in the neighbourhood. Selection of the weights is an important matter. More weight age is given to those pixels lying near to the point, near to the normal of the boundary and those lying on the boundary contours. Once a pixel is inpainted, it moves to next nearest pixel using weighted Bhattacharya Method. This ensures those pixels near the known pixels are inpainted first.

Super-Resolution (SR) is a class of techniques which refers to the process of creating one enhanced resolution image from one or multiple input low resolution images in an imaging system. The two corresponding problems are then referred to as single or multiple images SR, respectively. In both cases, the problem is of estimating high frequency details which are missing in the input image(s).

The proposed method builds upon the super-resolution based inpainting method proposed which is based on exemplar-based inpainting (in particular Criminisi like approach [5]) and single-image exemplar-based super resolution. The main novelty of the proposed algorithm is the combination of multiple inpainted versions of the input picture. The rationale behind this approach is to cope with the sensitivity of exemplar-based algorithms to parameters such as the patch size and the filling order. Different combinations have been tested and compared. Besides this major point, different adjustments regarding exemplar-based inpainting and SR methods are described such as the use of the coherence measure to constrain the candidate search.

In summary, the proposed method improves on the state of-the-art exemplar-based inpainting methods by proposing a new framework involving a combination of multiple inpainting versions of the input picture followed by a single-image exemplar-based SR method. Notice that the SR method is used only when the inpainting method is applied on a low resolution of the input picture.

There are many objectives and applications of inapaiting techniques. In photography and cinema, inpainting is used for restoration, to reverse the deterioration (e.g., cracks in photographs or scratches and dust spots in film) .It is also used for removing red-eye, the stamped date from photographs and removing objects to creative effect. 


\section{MethodologY}

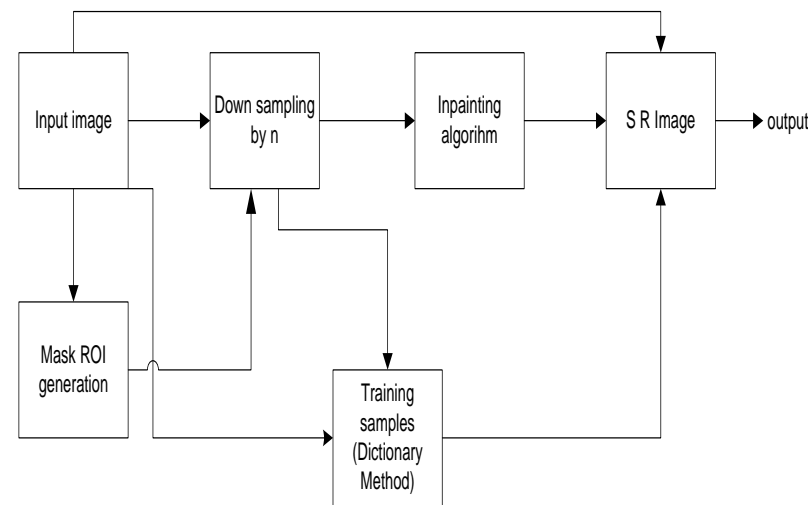

Figure 1: Block Diagram of the Proposed System

Figure1 illustrates the main concept underlying the proposed method. The two main components are the inpainting and the super-resolution algorithms. More specifically, the following steps are performed:

1. A low-resolution image is first built from the original picture;

2. An inpainting algorithm is applied to fill-in the holes of the low-resolution picture;

3. The quality of the inpainted regions is improved by using a single-image SR method.

The features used in this system are described as follows:

\section{A. Image Inpaintng}

In painting is the process of reconstructing lost or deteriorated parts of images and videos. For instance, in the museum world, in the case of a valuable painting, this task would be carried out by a skilled art conservator or art restorer. In the digital world, in painting refers to the application of sophisticated algorithms to replace lost or corrupted parts of the image data.

\section{B. Region of Interest}

A region of interest (ROI) is a selected subset of samples within a dataset identified for a particular purpose. For example: on a waveform (1D dataset), a time or frequency interval, on an image (2D dataset), the boundaries of an object etc. A ROI is a specification structure that allows for the definition of arbitrarily shaped regions within a given image, often called sub images (although a ROI can encompass the entire image if so defined). A ROI contains no image data. It is not an image itself, but a place holder that remembers a defined location within an image. ROIs can be irregular shapes, not necessarily the rectangle or square that our camera supplies. ROIs can be rectangles or squares (a square is often called an area of interest, or AOI), circles, annuluses, polygons and freehand shapes. Secondly, we may want to perform different image processing routines on different areas of an image, when we are only interested in a few small parts of it. Another reason for ROIs is that we can dynamically define and change them. We can draw a ROI over a portion of an image at run time, selecting their particular region of interest.

\section{Down sampling}

Down sampling is the process of reducing the sampling rate of a signal. This is usually done to reduce the data rate or the size of the data. The down sampling factor is usually an integer or a rational fraction greater than unity. This factor multiplies the sampling time or, equivalently, divides the sampling rate. The down sampled value is $4 .(M=4)$

Down sampling by an integer factor, $\mathrm{M}$ can be explained as a 2-step process, with an equivalent implementation that is more efficient:

- Reduce high-frequency components with a digital low pass filter.

- Decimate the output sequence, keeping only every $\mathrm{M}^{\text {th }}$ output sample.

Decimation causes high-frequency signal components to be misinterpreted by subsequent users of the data, which is a form of distortion called aliasing. The first step, if necessary, is to suppress such components to an acceptable level of distortion. In this application, the filter is called an anti-aliasing filter.

\section{Image restoration}

Image restoration is the operation of taking a corrupted/noisy image and estimating the clean original image. Corruption may come in many forms such as motion blur, noise, and camera miss focus. 


\section{E. Super Resolution}

Super resolution (SR) is a class of techniques that enhance the resolution of an imaging system. In some SR techniques - termed optical SR - the diffraction limit of systems is transcended, while in others-geometrical $\mathrm{SR}$ - the resolution of digital imaging sensors is enhanced.

\section{F. Super resolution algorithm}

Once the in painting of the low-resolution picture is completed, a single-image super-resolution approach is used to reconstruct the high resolution of the image. The idea is to use the low-resolution in painted areas in order to guide the texture synthesis at the higher resolution. The problem is to find a patch of higherresolution from a database of examples.

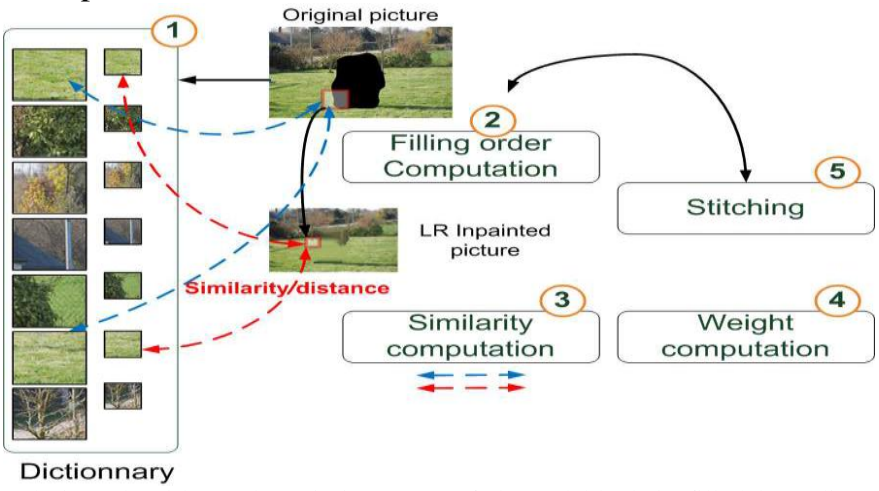

Figure 2: Flowchart of the super-resolution algorithm. The missing parts of the red block is filled by a linear combination of K HRcandidates (green arrows). The weights are computed using the similarity distance between LR and HR patches (green and red arrows, respectively). The top image represents the original image with the missing areas whereas the bottom one is the result of the low-resolution inpainting.

1. Dictionary building: it consists of the correspondences between low and high resolution image patches. The unique constraint is that the high-resolution patches have to be valid, i.e. entirely composed of known pixels. In the pro- posed approach, high-resolution and valid patches are evenly extracted from the known part of the image. The size of the dictionary is a user-parameter which might influence the overall speed/quality trade-off. An array is used to store the spatial coordinates of HR patches (DHR). Those of LR patches are simply deduced by using the decimation factor;

2. Filling order of the HR picture: the computation of the filling order is similar to the one described earlier. It is computed on the HR picture with the gradient-based method. The filling process starts with the patch having the highest priority. This improves the quality of the in painted picture compared to a raster-scan filling order;

3. For the LR patch corresponding to the HR patch having the highest priority, its K-NN in the in painted images of lower resolution are sought. The number of neighbours is computed using the nearest neighbouring pixels.

4. Weights $\mathrm{w}_{\mathrm{p}}, \mathrm{p}_{\mathrm{j}}$ are calculated by using a non-local means method as if we would like to perform a linear combination of these neighbours. However, the similarity distance used to compute the weights is composed of two terms: the first one is classical since this is the distance between the current LR patch and its LR neighbours. The second term is the distance between the known parts of the HR patch HRp and the HR patches corresponding to the LR neighbours of LRp. Say differently, the similarity distance is the distance between two vectors composed of both pixels of LR and HR patches. The use of pixel values of HR patches allows to constraint the nearest neighbour search of LR patches.

5. A HR candidate is finally deduced by using a linear combination of HR patches with the weights previously computed

$$
\psi_{p}^{H R}=\sum_{p_{j} \in \mathcal{D}^{H R}} w_{p, p_{j}} \times \psi_{p, p_{j}}
$$

6. Stitching: the HR patch is then pasted into the missing areas. However, as an overlap with the already synthesized areas is possible, a seam cutting the overlapped regions is determined to further enhance the patch blending. The minimum error boundary cut is used to find a seam for which the two patches match best. The similarity measure is the Euclidean distance between all pixel values in the overlapping region. More complex metrics have been tested but they do not substantially improve the final quality. At most four overlapping cases (Left, Right, Top and Bottom) can be encountered. There are sequentially treated in the aforementioned order. The stitching algorithm is only used when all pixel values in the overlapping region are known or already synthesized. Otherwise, the stitching is disabled.

After the filling of the current patch, priority value is recomputed and the afore-mentioned steps are iterated while there exist unknown areas. 


\section{Results}

In order to assess the performance of the proposed approach, the parameters of the algorithm are kept constant for the tests presented in this paper.

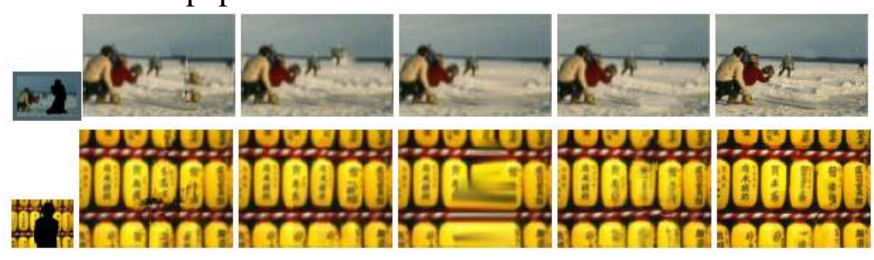

(a)

(b)

(c)

$(\mathrm{d})$

(e)

(f)

Figure 3: (a) low-resolution pictures with missing areas in black; (b) Criminisi et al.'s results; (c) Patch Match results; (d) Gradient -based results; (e) and (f) Proposed method (the down sampling factor is set to $n=4$; patch size is $11 \times 11$ ).

\section{Conclusion}

In this paper I have introduced a new inpainting framework which combines non-parametric patch sampling method with a super-resolution method. We first propose an extension of a well-known exemplerbased method (improvements are gradient-based priority, K-coherence candidates and a similarity metric and compare it to existing methods. Then, a super-resolution method is used to recover a high resolution version. This framework is interesting for different reasons. First the results obtained are within the state-of-the-art for a moderate complexity. Beyond this first point which demonstrates the effectiveness of the proposed method, this framework can be improved. For instance, one interesting avenue of future work would be to perform several inpainting of the low-resolution images and to fuse them by using a global objective function. First, different kinds of inpainting methods (patch-based or PDE-based) could be used to fill-in the missing areas of a lowresolution image. Second, for a given inpainting method, one can envision to fill-in the missing areas by using different settings e.g. for the patch size in order to better handle a variety of textures and to better approach the texture element sizes. Finally, we believe that the proposed framework will be appropriate for video completion. This application is indeed very time-consuming. The use of the proposed framework could dramatically reduce the computational time.

\section{ACKNOWLEDGMENT}

I wish to express my sincere gratitude to my guide Ms. Preetha D Souza for her valuable guidance and timely suggestions and also to Prof.B.V.Nityananda, Head of the Department, Dept. of Electronics \& Communication and also to my Principal Dr. Joseph Gonsalvis, St. Joseph Engineering College, Vamanjoor, Mangalore, for his support and encouragement.

\section{REFERENCES}

[1] Ashikhmin, M, “Synthesizing natural textures”, In I3D’01. (2001).

[2] Bertalmio, M., Sapiro, G., Caselles, V., Ballester, C. "Image inpainting", In SIG-GRAPH 2000. (2000).

[3] Chan, T., Shen, J "Variational restoration of non-flat image features: models and algorithms", SIAM J. Appl. Math. 61 (2001) 13381361 .

[4] Chang, H., Yeung, D.Y., Xiong, Y., "Super-resolution through neighbor embedding”, In Computer Vision and Pattern Recognition Volume I. (2004) 275-282.

[5] Criminisi, A., P'erez, P., Toyama, K, "Region filling and object removal by examplar- based image inpainting", IEEE Trans. On Image Processing 13 (2004) 1200-1212.

[6] Efros, A.A., Leung, T.K, "Texture synthesis by non-parametric sampling”, In International Conference on Computer Vision. (1999) 1033-1038.

[7] Freeman,W.T., Jones, T.R., Pasztor, E.C, "Example-based super resolution”, IEEE Computer Graphics and Applications 22 (2002) 5665.

[8] Glasner, D., Bagon, S., Irani, M., "Super-resolution from a single image". In 2009 IEEE 12th International Conference on Computer Vision (ICCV). Volume 10.(2009) 349356

[9] Tschumperl'e, D., Deriche, R.,"Vector-valued image regularization with pdes", a common frame work for different applications. IEEE Trans. On PAMI 27 (2005) 506-517.

[10] Dai, S., Han, M., Xu, W., Wu, Y., Gong, Y., Katsaggelos, A Softcuts: “a soft edge smoothness prior for color image superresolution". IEEE Trans. On Image Processing 18 (2009) 969-981.

[11] Kopf, J., Kienzle, W., Drucker, S., Kang, S.B. "Quality prediction for image completion", ACM Transactions on Graphics (Proceedings of SIGGRAPH Asia 2012) 31 (2012)

[12] Le Meur, O., Guillemot, C.: Super-resolution-based inpainting. In ECCV. (2012)

[13] Xu, Z., Sun, J. "Image inpainting by patch propagation using patch sparsity”. IEEE Trans. on Image Processing 19 (2010) $1153-1165$ 